
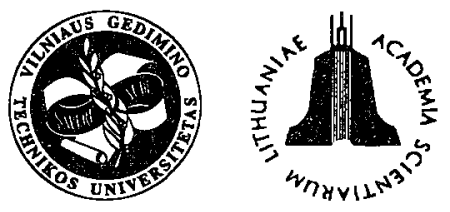

ISSN 1648-4142 TRANSPORT

http:/www.vtu.lt/english/editions

\title{
FORMULATION OF OPTIMAL INTERNATIONAL FREIGHT TRANSPORT OBJECTIVE
}

\author{
Aldona Jarašūnienè \\ Vilnius Gediminas Technical University, Transport Research Institute, \\ Plytines g. 27, LT-2040 Vilnius, Lithuania \\ e-mail: ajarasuniene@yahoo.com
}

Received 200206 01; accepted 20020910

\begin{abstract}
To increase the attraction of Lithuania as a transit country striving to promote carriers' border crossing activities and facilitate customs clearance procedures as well as freight delivery to clients it is necessary to identify the main obstacles, to analyse them and to select adequate measures and means for their elimination.

Therefore, on the basis of the formulation of transport freight management objective, as well as basing on the assessment of indeterminacy of external impacts, it would be possible to deduce the main causes of idle time of transport means in customs, to estimate the dependence of service time in proportion to transport flow.
\end{abstract}

Keywords: transport flow, management of transport flows, theoretical research, idle time of transport means.

\section{Introduction}

Transport activities in Lithuania make a large share of the Gross Domestic Product (GDP). Transit transport contributes its significant share to GDP as well. With the aim to increase the attraction of Lithuania as a transit country it is necessary to identify the main obstacles in the field of border crossing and formalities of customs procedures.

Analysing this situation it is important to explore the incoming and outgoing transit transport flows, to identify their theoretical distribution in proportion to customs and transport pressure on customs as well, to formulate the objectives of management of transport flows and to assess the indeterminacy of external impacts.

The analysis of freight flows enables the elaboration of recommendations for the improvement of customs inspection by the introduction of "green lanes" for cars carrying freights of certain groups, thus diminishing to minimum the idle time in customs. The analysis of freight structure and distribution according to consignor and consignee countries enables significant influencing of incoming, outgoing and transit freight flows per each customs house. On this basis it is possible to identify measures for solving the problems of the busiest customs-houses, thus positively influencing Lithuanian economy and enabling a more precise view of inspection of customable freight carrying transport means' with the present number of customs officials.

Basing on the formulation of objectives of management of transport flows and on the assessment of indeterminacy of external impacts it will be possible to perform detailed research of flow distribution of incoming transport means according to the time and their distribution in sepa- rate customs lanes [1-8]. It will also allow to identify the idle time of transport means and to measure the dependence of carriers' service time in proportion to transport flow.

\section{Management of Transport Flows}

Making the analysis of management of transport flows it is presumed that transport flows are moving in the given network $G=(V, E)$ which has $n$ peaks and $m$ panes. In the network the beginning of flow at the peak 1 and the end of flow at the peak $n$ are fixed. The traffic of the flow along each pane lasts one time interval. The time is discrete and has only positive meanings $t=0,1, \ldots, T$, here $T$ - period of management (planning).

At each time moment $t$ the state of the process of movement of flow is characterised by $n$ phasic coordinates $x_{1}(t), \ldots, x_{n}(t)$, here $x_{i}(t)$-the amount of the flow remaining on the peak $i$ at the time interval $[t, t+1]$. Thus at every moment $t$ the phasic state $X(t)$ has $n$ coordinates:

$$
X(t)=\left(x_{1}(t), x_{2}(t), \ldots, x_{n}(t)\right) .
$$

The object state sequence $X(0), X(1), \ldots, X(T)$ at the moments $t=0,1, \ldots, T$ is called the trajectory of the movement of the object.

We will manage the flow movement at each time $t$ moment selecting $m$ managing parameters

$U(t)=\left(u_{1}(t), \ldots, u_{n l}(t)\right.$, here $u_{j}(t)$-amount of flow starting to move along the pane $j$ at $t$ time moment.

The sequence of points is called the management 
$U(1), U(2), \ldots, U(T)$. We introduce restrictions to management field and to phasic coordinates.

It is considered that the possibilities of the pane capacity and capacity of stores at the peaks are the functions of the time $t$ and incidental factor $\xi$. Let us indicate:

$$
f(t, \xi)=\left(f_{1}(t, \xi), \ldots, f_{m}(t, \xi)\right)
$$

and

$$
l(t, \xi)=\left(l_{1}(t, \xi), \ldots, l_{m}(t, \xi)\right) .
$$

Accordingly the vectors of capacity possibilities and the lower margin at the moment $t$, specifying a permissible management field, and

$$
g(t, \xi)=\left(g_{1}(t, \xi), \ldots, g_{n}(t, \xi)\right)
$$

store capacity vector specifying restrictions to phasic coordinates.

Then, in the network of transport flows, we shall formulate the optimal management issue as the stochastic issue of optimal management of discrete object:

$$
\begin{aligned}
& J_{0}(X, U, t) \rightarrow \max ; \\
& \Delta X(t)=\varphi(U, t), t=\overline{0, T-2} ; \\
& l(t, \xi) \leq U(t) \leq f(t, \xi), t=\overline{0, T-1} ; \\
& X(T) \leq g(t, \xi), t=\overline{1, T} ; \\
& \left.\begin{array}{l}
X(t) \geq 0 \\
U(t) \geq 0
\end{array}\right\}, \quad t=\overline{0, T} .
\end{aligned}
$$

And the set of initial states $X(0)$ - is put:

$$
X(0)=X^{0} \text {, }
$$

here (1) - criterion of management efficiency;

(2) - management of the movement of a managed object;

(3) - permissible management range;

(4) - limitations for phasic coordinates;

(5) - non-negativity of phasic variable and managing parameters.

By the stochastic model (1)-(5) it is possible to define a great class of transport flows optimal management objectives.

Let $\beta\left|b_{i j}\right|$ be the transport network $G$ matrix of peakpane incidences, whereas $b_{i j}=-1$, if the pane $j$ goes out from the peak $i$ and $b_{i j}=+1$, if the pane $j$ goes into the peak $i$. The matrix $\beta^{+}$is obtained also changing the negative elements of $\beta$ by zeroes, and $\beta$ - changing positive elements with zeroes. Then discrete managed object which is defined by the conditions (1)-(5) will be described in the network of dynamic flow in proportion to the respective parameters:

$$
\begin{aligned}
& X(t+1)=X(t)+B^{+} U(t)+B^{-} U(t+1), t=\overline{0, T-2} \\
& X(T)=X(T-1)+B^{+} U(T-1) \\
& X(0)=X^{0} \\
& l(t, \xi) \leq U(t) \leq f(t, \xi)
\end{aligned}
$$

$$
\begin{aligned}
& X(t) \leq g(t, \xi), t=\overline{1, T} ; \\
& \left.\begin{array}{l}
0 \leq X(t) \\
0 \leq U(t)
\end{array}\right\}, \quad t=\overline{0, T} .
\end{aligned}
$$

Here we will present four most typical objectives of dynamic management of transport flows.

1. The objective of maximum dynamic flow in the network. It is necessary to maximise quantities of the flow delivered to the destination point per $T$ time moments. Formally the essence of the objective is that knowing the initial state (8) we chose such permissible management (9), (11) for the object $(6,7,10,11)$, which gives maximum meaning to the functional $J_{0}(X, U, t)$. In this issue the criterion is:

$$
X_{n}(T) \rightarrow \max .
$$

Initial conditions in this issue will be presumed in the following shape $X^{0}=(\infty, 0, \ldots, 0)$. Sometimes additional conditions are assumed that the whole flow going out from the peak 1 will reach the destination peak $n$ at $T$ time periods. To fulfil this condition we shall presume $g(T)=\{\infty, 0, \ldots, 0, \infty\}$.

2. The objective of minimal cost. Let $R(t)$ be the cost vector of carrying of a flow unit along the network panes at the time moment $t$. It is necessary to minimise the notion:

$$
\sum_{t=0}^{T=1}(R(t), U(t)) \rightarrow \min
$$

limitations being $(6,11)$.

Initial conditions have the shape:

$$
X^{0}=(x, 0, \ldots, 0),
$$

here $x$ - the extent of flow to be carried along the network $G$. In order that the whole flow could reach the peaks $n$, it is necessary that:

$$
g(T)=\{0,0, \ldots, 0, x\} .
$$

Without this precondition it may be stated that:

$$
g(T)=\left(0, \alpha_{2}, \alpha_{3}, \ldots, \alpha_{n}\right),
$$

when $0 \leq \alpha_{i} \leq x, \sum \alpha_{i}=x$.

3. Minimisation of the flow extent filling in the $d y$ namic set of panes. Let us select in the network $G$ the minimum extent dynamic flow from 1 to $n$ at $T$ intervals time filling in the panes from $\Omega(t)$ and meeting the conditions (6)-(11). The criterion of the objective is as follows:

$$
X_{n}(T) \rightarrow \max ,
$$

whereas $l(t ; \xi)=f(t ; \xi)$ for all panes from the set $\Omega(t)$. Initial conditions are:

$$
\begin{aligned}
& X^{0}=(\infty, 0, \ldots, 0) \text { and } \\
& g(T)=(\infty, 0, \ldots, 0, \infty)
\end{aligned}
$$

According to the contents this issue is interpreted as an objective of identification of a minimal number of transport means with the aim of ensuring the transport traffic according to the schedule, organising the carriage 
by all transport modes except pipeline transportation.

4. Maximisation of transportation profit. Let us presume the given flow extent $x$, which is at a time moment in 0 resource. For each pane we will introduce two extents

$$
f_{e}^{1}(t, \xi), f_{e}^{1}(t, \xi), f_{e}^{2}(t, \xi)=f_{e}^{1}(t, \xi),+f_{e}^{2}(t, \xi)
$$

Let us presume that the flow along the pane consists of two parts $U_{e}^{1}(t)$ and $U_{e}^{2}(t)$. To the flow $U^{1}(t)$ we shall relate the cost amount

$\left(R(t), U^{1}(t)\right)$, to $U^{2}(t)$ - the profit amount $\left(C(t), U^{2}(t)\right)$ when traffic is going along the network panes.

It is necessary to make a dynamic flow of a given extent in the network $G$, maximising general transportation profit during $T$ time moments.

Criterion of the objective:

$$
\begin{aligned}
& \sum_{t=0}^{T=1}\left[\left(C(t), U^{2}(t)\right)\left(R(t), U^{1}(t)\right)\right] \rightarrow \max \\
& U^{1}(t)+U^{2}(t)=U(t) .
\end{aligned}
$$

Initial conditions are the same as of the second objective.

$$
\begin{aligned}
& 0 \leq U^{1}(t) \leq f^{1}(t, \xi), \quad t=\overline{0, T-1} . \\
& 0 \leq U^{2}(t) \leq f^{2}(t, \xi)
\end{aligned}
$$

The fourth objective is peculiar for the planning of freight transportation by the maritime transport. The profit is related to the freight transportation and the costs - to the transportation of ballast.

\section{Assessment of the Indeterminacy of External Impact}

Let us analyse the objective of the optimal management of transport flows (1)-(5).

Since the management model (3)-(4) contains random factors, it cannot be stated that the functional $J_{0}$ is determined extent and it is necessary to analyse it as a probability characteristics of a random/incidental process (mathematical expectancy, dispersion, probability of reaching a fixed state).

For the solution of the objective we shall use a two stage scheme of optimisation. Here in the first stage it is stated that $\xi=0$ and the solution is sought of a determined objective (programme trajectory), but in the second stage it is considered that $\xi$ is a small deviation and a minimisation objective of the occurring deviation from the programme trajectory is solved.

Thus for the search of the programme solution the initial objective will be written as follows:

$$
\begin{aligned}
& J_{0}(X, U, t) \rightarrow \max \\
& \Delta X(t)=\varphi(U, t) ; \\
& l(t) \leq U(t) \leq f(t) ; \\
& X(t) \leq g(t) .
\end{aligned}
$$

The solution of this discrete optimal management objective may be obtained using the discrete maximum principle or traditional methods of network solution which as a rule tend towards the solution of a static objective in a relevant deployed network.

Let us presume that the solution of the objective (17) has been obtained and it is of the following shape $\widetilde{X}(t)$ and $\tilde{U}(t)$. Now, assuming that interferences $\xi$ are sufficiently insignificant, we shall seek for the solution of the objective (1)-(5) in the shape of:

$$
\begin{aligned}
& X(t)=\tilde{X}(t)+y(t) \\
& U(t)=\tilde{U}(t)+v(\hat{t})
\end{aligned}
$$

here $y(t)$ and $v(t)$ are small successions as well as in $\xi$. Having inserted (6) into the initial issue (1)-(5) and having deployed the functions $\varphi, l$ and $f$ into the line according to $y, v, \xi$ and leaving only linear members we shall obtain:

$$
\begin{aligned}
& \Delta y(t)=a(t) v(t) \\
& y(t)=g(t) \xi+g(t) ; \\
& l(t)+\theta(t) \xi \leq v(t) \leq \delta(t) \xi+f(t) ; \\
& \bar{g}(t)=g(t)-\tilde{X}(t) ; \\
& \bar{f}(t)=f(t)-\tilde{U}(t) ; \\
& l(t)=l(t)-\tilde{U}(t) ; \\
& \alpha=\left(\frac{\partial \varphi}{\partial \xi}\right) \quad \theta=\left(\frac{\partial l}{\partial \xi}\right) ; \\
& \gamma=\left(\frac{\partial g}{\partial \xi}\right) \quad \delta=\left(\frac{\partial f}{\partial \xi}\right) .
\end{aligned}
$$

Here all the derivative programme trajectories are calculated along the system (i. e. at the zero meanings of $\xi, v, y$ ). In the second stage for various objectives the functional, which has to be minimised, may be of different expression. In general shape we shall put it as follows:

$$
J_{1}(y, v, t) \rightarrow \min .
$$

Further, in the second stage, we shall solve the objective identifying the correcting management $v(x, t)$ and the reaching minimum to functional (20) to the conditions (19). For finding $v(x, t)$ it is necessary to know how to measure present solution diversions from that of the programme, at least at relevant discrete moments and to select correcting impacts according to the results of this measurement. The objective is solved by the mechanism of reversible synthesis. However, in general case, there are no regular methods of making the reversible mechanism. We shall apply the above said general solution sequence for the $1-4$ objectives. After restoring (18) into the conditions (6)-(11) and after linerisation, we shall obtain:

$$
\begin{aligned}
& y(t+1)=y(t)+B+v(t)+B-v(t+1) ; \\
& y(T)=y(T-1)+B+v(T-1) ;
\end{aligned}
$$




$$
\begin{aligned}
& y(0)=0 ; \\
& \bar{l}(t)+\theta(t) \xi \leq v(t) \leq \delta(t) \xi+\bar{f}(t) ; \\
& y(t) \leq \gamma(t) \xi+g(t) .
\end{aligned}
$$

Further we shall state that the quantities $\theta(t)$ and $\delta(t)$ for each $t$ cannot have a meaning with various symbols and the quantities $\theta(t) \xi, \delta(t) \xi$ and $\gamma(t) \xi$ may be positive and negative as well.

Let us take the criteria of the second stage for the objectives 1-4.

1 Objective.

$y_{n}(T) \rightarrow \min$.

2 Objective.

$$
\left|\sum_{t=0}^{T}(R(t), v(t))\right| \rightarrow \min .
$$

3 Objective.

$Y_{n}(T) \rightarrow \min$.

if $\theta(t)=\delta(t)$, for all branches of a lot $\Omega(t)$.

4 Objective.

$$
\begin{aligned}
& \left.\mid \sum_{t=0}^{T-1}\left[\left(C(t), v^{2}(t)\right)-(R(t), v \circlearrowleft(t))\right]\right] \rightarrow \min ; \\
& v^{1}(t)+v^{2}(t)=v(t) ; \\
& v^{1}(t) \leq \delta^{1}(t) \xi, v^{2}(t) \leq \delta(t) \xi .
\end{aligned}
$$

Further, basing on theoretical research, it would be possible to make the analysis of the distribution (according to time) of flows of cars entering a customs post as well as the analysis of their distribution into separate customs lanes. It would also be possible to identify the idle time of transport means caused by customs procedures, to identify the dependence of the time of services given to carriers in proportion to the amount of transport flow, to calculate and to assess the optimal selection of transport amount for inspection in customs during a certain period of time.

\section{Practical Assessment of the Theoretical Research of International Freight Transport Flows}

The theoretical research of international freight transport flows allows to make a detailed analysis of freight flows enabling the elaboration of the recommendations for the improvement of customs inspection procedures. Basing on this the analysis of freight structure and distribution according to countries would enable to prepare the most influential forecasts of transit freight transport by separate customs-houses.

The stochastic management model obtained as a result of the theoretical research of international freight transport flows enables the determination of a great group of optimal international freight flows management issues, such as the issue on maximum dynamic flow in the network, which would allow to calculate maximum transport rate in a cus- toms post; the objective of minimum cost, defining the extent of transported flow filling in the dynamic set of the pane, the objective the essence of which is the determination of minimum quantity of international transport means allowing efficient scheduling of transport traffic.

\section{Conclusions}

1. Aiming to enhance Lithuania's attraction as a transit country it is necessary to identify the most important obstacles and after their analysis to select means and measures for their elimination.

2. It is important to define and to analyse transit transport flows, to identify their theoretical distribution in proportion to customs-houses and to investigate how busy they are with transport means.

3. On this basis it is possible to identify measures and means of solution of the busiest customs-houses, thus enabling positive impact to Lithuanian economy, as well as to make more relevant assessment of the inspection of transport means (carrying customable freight) performed by a present number of staff.

4. Basing on the assessment of formulation of the objectives of management of transport flows and on the indeterminacy of external impacts, it is possible to carry out a more detailed analysis of the distributions (according to time) of flows of transport means entering customs, as well as their distribution in to separate customs lanes. It also enables the definition of dependency on the extent of transport flow, as well as allows the calculation of choosing optimum transport amount for the inspection in customs-houses during a certain period of time.

\section{References}

1. Frank Southworth, Bruce E.Peterson. Intermodal and intermodal freight network modelling. Transportation $R e$ search, Part C 8, 2000, p 147-166.

2. Powell Warren B. Carvalko Tassio, A Real-Time Optimisation of Containers and Flatcars for intermodal Operations. Transportations Science, Vol 32, No 2, May 1988, p 110-126.

3. Zhan F.-B., Noon Ch.-E. Shortest Path Algorithms: An Evaluation using Real Road Networks. Transportation Science, Vol 32, No 1. February, 1988, p 65-73.

4. Nuzzolo A., Russo F., Cristali U. A Doubly Dynamic Schedule - based Assignment Model for Transit Networks. Transportation Science, Vol 35, No 3. August, 2001, p 268-285.

5. Gattorna D. W. Walters. Managing the Supply Chain. A Strategic Perspective. Macmillas Press Ltd., 1998. 310 p

6. Coule J. Bordi E., Carinato J. Transportations Third edition. USA, 1999. $525 \mathrm{p}$

7. Transport of fast Changing Europe. Vers un Reseau European des systems de transport by Group Transport 2000 Plus. Brussels, 2000. $90 \mathrm{p}$

8. Baublys A. Introduction to the theory of transport system. (Transporto sistemos teorijos ivadas). V.: Technika, 1997. 298 p (in Lithuanian). 